ARTICLE

https://doi.org/10.1038/s41467-019-09225-7

OPEN

\title{
Intrinsic photoisomerization dynamics of protonated Schiff-base retinal
}

Hjalte V. Kiefer (1) ${ }^{1}$, Elisabeth Gruber (1) ${ }^{1}$, Jeppe Langeland', Pavel A. Kusochek ${ }^{2}$, Anastasia V. Bochenkova (i) ${ }^{2}$ \& Lars H. Andersen (1) ${ }^{1}$

The retinal protonated Schiff-base (RPSB) in its all-trans form is found in bacterial rhodopsins, whereas visual rhodopsin proteins host 11-cis RPSB. In both cases, photoexcitation initiates fast isomerization of the retinal chromophore, leading to proton transport, storage of chemical energy or signaling. It is an unsolved problem, to which degree this is due to protein interactions or intrinsic RPSB quantum properties. Here, we report on time-resolved actionspectroscopy studies, which show, that upon photoexcitation, cis isomers of RPSB have an almost barrierless fast 400 fs decay, whereas all-trans isomers exhibit a barrier-controlled slow 3 ps decay. Moreover, formation of the 11-cis isomer is greatly favored for all-trans RPSB when isolated. The very fast photoresponse of visual photoreceptors is thus directly related to intrinsic retinal properties, whereas bacterial rhodopsins tune the excited state potentialenergy surface to lower the barrier for particular double-bond isomerization, thus changing both the timescale and specificity of the photoisomerization.

\footnotetext{
${ }^{1}$ Department of Physics and Astronomy, Aarhus University, 8000 Aarhus C, Denmark. ${ }^{2}$ Chemistry Department, Lomonosov Moscow State University, 119991 Moscow, Russia. Correspondence and requests for materials should be addressed to L.H.A. (email: Iha@phys.au.dk)
} 
B acteriorhodopsin (bR) is a seven helix membrane protein employed by halobacteria as a proton pump to convert light into chemical energy by transporting protons across the cell membrane ${ }^{1,2}$. It contains the all-trans retinal chromophore in the protonated Schiff-base form (RPSB), which isomerizes upon photoexcitation from its native all-trans to the 13-cis form with high specificity $(64 \%)$ and speed $(500 \mathrm{fs})^{3-5}$. According to the current belief, interactions within the protein facilitate isomerization that takes place with a speed about ten times faster than encountered without such interactions since isomerization takes about $2-10$ ps in solutions ${ }^{4,6,7}$. In solutions, both 11 -cis and 13-cis products are formed, with total quantum yields on the order of $10-30 \% 4,6,8,9$, and hence high specificity is obtained in proteins and not in solutions. It is also known that the retinal chromophore isomerizes upon photoexcitation in the gas phase; however, the efficiency as well as speed are unknown ${ }^{10,11}$.

Another class of retinal proteins is rhodopsins, responsible for the primary event in vision ${ }^{12}$, and having the 11-cis RPSB chromophore. Experimental ${ }^{13-18}$ and theoretical ${ }^{19-21}$ studies have shown that the 11-cis to all-trans isomerization in rhodopsins is extremely fast and likely caused by dynamics on an almost barrierless potential energy surface of the first $\left(S_{1}\right)$ excited state 22,23 . The progression from the initially populated Franck-Condon point to a fluorescent state in $S_{1}$ takes, for example, $<100 \mathrm{fs}^{16}$, and full isomerization through a conical intersection is completed within $200 \mathrm{fs}^{13,14,19}$. Rhodopsins also show high $65 \%$ quantum yields for isomerization to all-trans. The cis isomer is found to behave much faster than all-trans in $\mathrm{MeOH}$ at early times, but showed the same slow (4 ps) behavior on the longer timescale $24-26$. To account for both fast and slow dynamics, as well as the low quantum yield of only 0.22 in solution, it was proposed that a primary role of the protein is to adjust the ratio between ground-state chromophore conformations that lead to reactive and nonreactive decay channels ${ }^{27}$.

The two classes of proteins with all-trans and 11-cis RPSB chromophores both exhibit ultrafast sub-ps excited-state dynamics in their host proteins, yet their dynamics in solutions is multi-exponential and shows a long ps component. Interestingly, the lifetime of all-trans RPSB in solutions may be shortened by an order of magnitude from $\sim 4$ to $\sim 400 \mathrm{fs}$ upon chemical modifications of the retinal backbone with a wide spread in quantum yields ${ }^{28,29}$. The most likely explanation points towards tuning of the excited-state potential-energy surface around one or more conical intersections. These are notoriously hard to characterize theoretically, and electron correlation as well as nuclear dynamics must be treated accurately ${ }^{30}$. Based on QM/MM calculations of an RPSB model chromophore, it was proposed that the excitedstate lifetime difference obtained in isolation and in $\mathrm{MeOH}$ may be traced to isomerization barriers primarily due to electrostatic interactions in the solution phase ${ }^{23}$.

Years of studies have been concerned with the related subject of spectral tuning (opsin shift) in proteins, where the band maximum traditionally has been compared to that obtained in $\mathrm{MeOH}$ solution $(440 \mathrm{~nm})^{8}$. This value is far from the intrinsic transition wavelength of about $610 \mathrm{~nm}$ obtained in the gas phase for both all-trans and 11-cis RPSB chromophores ${ }^{31-33}$, as well as those found in retinal-containing proteins. Interactions inside proteins, as well as in solutions, hence significantly change the energy gap between the ground and excited electronic states in the Franck-Condon region, and may also change the excited-state potential-energy surface around conical intersections. This may cause optimum conditions for fast isomerization and high quantum yields in proteins. Alternatively, the difference may be ascribed to significant perturbations taking place primarily in solutions, which slow down the isomerization and change the quantum yield significantly.
Despite numerous and advanced time-resolved studies of retinal proteins and RPSB chromophores in solutions, reference measurements obtained in the gas phase without solvent effects (H-bonding, steric interactions, electric fields, counterions, etc.) have not been obtained. Such measurements are needed to access the exact role of a hosting medium with respect to key properties such as quantum yields and excited-state lifetimes, which in turn are also related to fluorescence properties $25,34,35$. The reason for this situation is the tremendous experimental challenge encountered when working with extremely dilute clouds of ions in the gas phase, where the chromophore density is orders of magnitude smaller than in solutions. Despite the challenges, such data may provide a stringent test of ab initio theories, which must be able to satisfactorily deal with the chromophore in isolation before any predictions of transition energies, lifetimes, and quantum yields in environments may be considered fully reliable.

Here, we report on an experimental excited-state dynamics study of the RPSB chromophores in isolation (gas phase). A new experimental approach, combining time-resolved action spectroscopy ${ }^{36}$ with femtosecond pump-probe techniques ${ }^{37}$ in an ionstorage ring, is used. The new technique is applicable to anions ${ }^{36}$ as well as cations, such as the retinal protonated Schiff-base chromophore. The studies include variations of key parameters, such as the pump and probe wavelengths and the chromophoreion temperature. High-level ab initio calculations are performed to support our conclusions.

\section{Results}

Pump-probe measurements. The experiments are performed at the ion-storage ring SAPHIRA ${ }^{38}$ (Fig. 1a), where RPSB chromophore ions (Fig. 1b) are stored in vacuo for several milliseconds. The absorption of photons is registered by the induced fragmentation and subsequent detection of neutral photofragments (Fig. 1c, d). The rate of statistical fragmentation (action) depends on the total internal energy of the ions, and therefore on the number of photons absorbed ${ }^{39}$. From the fragmentation rate, we are able to deduce when, after initial $S_{0} \rightarrow S_{1}$ excitation, a second probe photon of given energy and delay may be absorbed. This has been utilized in two different pump-probe schemes to obtain the excited-state decay as well as the ground-state recovery.

The present new approach of two-dimensional time-resolved action spectroscopy works as follows. A pump pulse first excites the RPSB chromophore in the broad $\mathrm{S}_{0} \rightarrow \mathrm{S}_{1}$ absorption band, which in the gas phase is between 550 and $650 \mathrm{~nm}^{31-33}$. To follow the evolution in the excited state, a probe wavelength of either 800 or $900 \mathrm{~nm}$ was used. At these wavelengths, subsequent $S_{1} \rightarrow S_{n}$ excitation may take place ${ }^{33}$ causing fast statistical fragmentation of the chromophore ions after sequential absorption of two photons (pump and probe) and internal conversion to the $S_{0}$ ground state. The ground state is not re-excited at these probe wavelengths, and hence the fast two-photon fragmentation signal disappears as soon as the system has left the $S_{1}$ excited state and returned to $S_{0}$ (Fig. $2 \mathrm{a}, \mathrm{b}$ ). In contrast, when a probe wavelength, resonant with the $S_{0} \rightarrow S_{1}$ electronic transition is used, one retrieves the ground-state recovery after internal conversion by the growing fast, two-photon action signal with increasing pumpprobe delay (Fig. 2c, d). Identification of the absorption of two consecutive photons is provided by the time at which the molecular dissociation takes place, hence time in two dimensions: the statistical fragmentation time and the pump-probe delay.

The time-resolved pump-probe fragmentation signal with 580 $\mathrm{nm}$ pump pulses and $800 \mathrm{~nm}$ probe pulses is shown in Fig. 2a. A fast decay is observed when the $580 \mathrm{~nm}$ pump comes prior to the $800 \mathrm{~nm}$ probe (positive delay). Here the RPSB chromophore is first excited to $S_{1}$ by the pump pulse, and further excited to $S_{3}$ by 

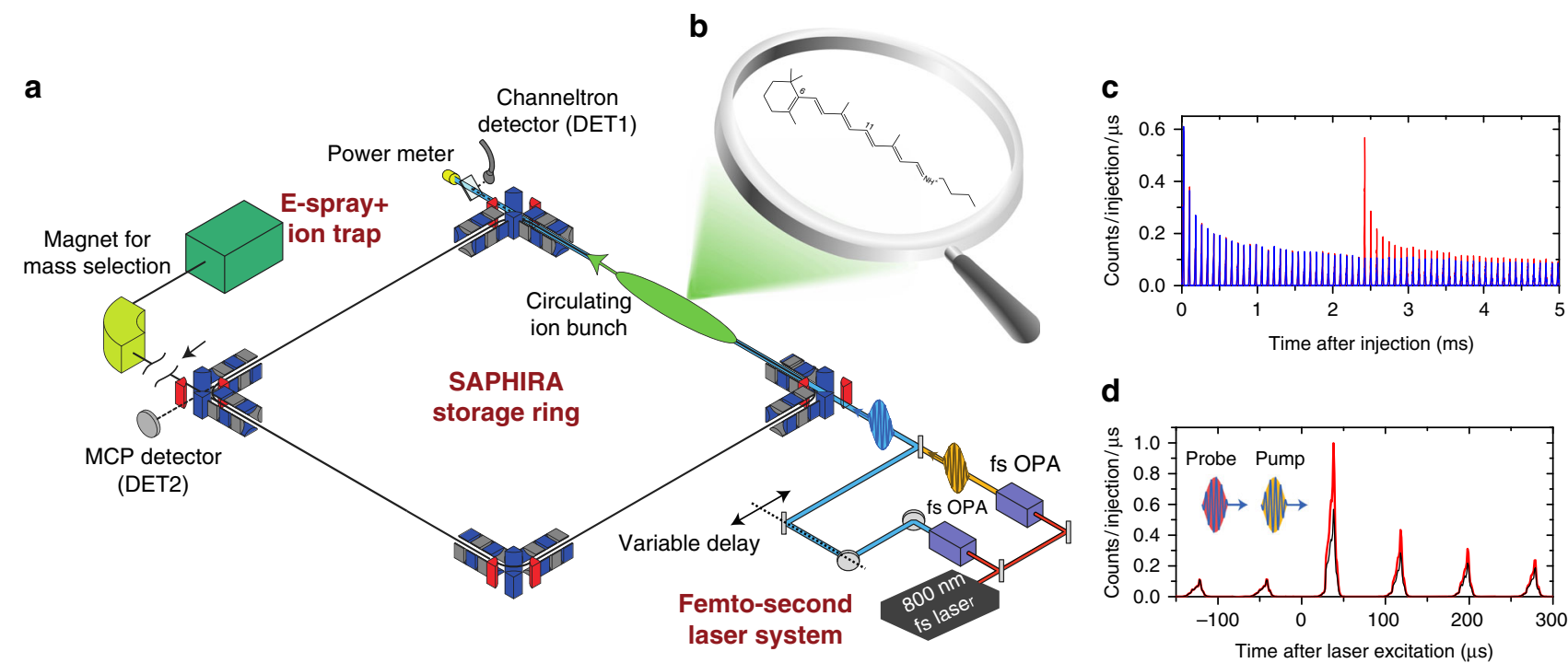

Fig. 1 Experimental technique. a The experimental setup at the electrostatic ion-storage ring SAPHIRA 38 . Ions are produced by electrospraying retinal chromphores dissolved in methanol. The chromophore ions are stored in SAPHIRA before being irradiated by femtosecond laser pulses. $\mathbf{b}$ The all-trans protonated Schiff-base retinal chromophore. c Circulating ions produce neutral fragments from collisions with the residual gas (blue curve). Absorption of photons (after $2.4 \mathrm{~ms}$ of ion storage) increases the internal energy and causes statistical fragmentation after internal conversion to the ground state, as measured by the micro-channel plate (MCP) detector (red curve) ${ }^{39}$. d At each pump-probe delay laser-induced fragmentation is registered as a function of storage time in SAPHIRA. The pump-probe signal is obtained by subtracting background contributions (collisional background as well as single-pulse events) from the total two-pulse signal (see Supplementary Figure 1)

the $800 \mathrm{~nm}$ probe pulse $\mathrm{e}^{33}$. No fast, two-photon, action is observed when the pulses arrive in the reversed order (negative delay) due to the lack of ground-state excitation with $800 \mathrm{~nm}$ pulses. The signal also disappears at late times when the ground state is repopulated after internal conversion. The data show two lifetimes of approximately $400 \mathrm{fs}$ and 3 ps, with an ultrafast sub$100 \mathrm{fs}$ component, revealed from a three-exponential fit (Fig. 2a inset), ascribed to wavepacket dynamics in the Franck-Condon region. Similarly, when the probe-pulse wavelength is set to probe the same $S_{0}$ to $S_{1}$ transition as the pump pulse (580 nm, Fig. $2 \mathrm{c}$ ), the two-photon absorption signal appears when the $S_{0}$ ground state becomes repopulated with the same set of lifetimes. The two lifetimes were found to be insensitive to the choice of wavelength (see Supplementary Figures 2 and 3).

In Fig. 2e, we consider the case with 580 and $400 \mathrm{~nm}$ pulses. Both are capable of exciting the ground state (to $S_{1}$ and $S_{2}$, respectively), though with different efficiency. This gives a signal at asymptotic negative as well as positive delay times. The $\mathrm{S}_{0} \rightarrow \mathrm{S}_{2}$ transition has a low oscillator strength, and the $400 \mathrm{~nm}$ probe pulse is absorbed from the $S_{1}$ state more efficiently than from $S_{0}$ after internal conversion ${ }^{33}$, resulting in the overall decay of the signal at positive delay times. Notably, when the RPSB is excited to $S_{2}$ by $400 \mathrm{~nm}$ excitation, it returns to the ground state in ca. $500 \mathrm{fs}$, as evidenced from the dynamics at negative delay times. Such fast relaxation of RPSB in $\mathrm{S}_{2}$ was also seen in fluorescence studies of RPSB ions in solvents ${ }^{35}$. However, more detailed gasphase studies are required to fully resolve the relaxation dynamics of RPSB following its excitation to $\mathrm{S}_{2}$.

The temperature of the chromophore ions prior to injection into the ion-storage ring may be controlled by cooling the ion trap inside the ion source with liquid nitrogen. At $100 \mathrm{~K}$, the lifetimes are found to increase significantly to 1.4 and $77 \mathrm{ps}$, as shown in Fig. 3 (compared to $400 \mathrm{fs}$ and $3 \mathrm{ps}$ at $300 \mathrm{~K}$ ). This indicates that both lifetime components are controlled by one or more barriers in the excited state.

It is in general challenging to completely isolate all-trans from single-cis isomers of RPSB in the gas phase as well as in solutions, where exposure to light generates isomerized forms that are nonstatistically populated $32,40,41$. Ion mobility measurements of the RPSB chromophore have shown an almost equal content of alltrans versus single cis isomers after electrospraying the all-trans isomer ${ }^{41}$. Based on these results, we might expect a significant fraction of the ions populated in single cis isomers, separated from the all-trans configuration by relatively high barriers. We therefore consider both all-trans and single cis isomers as relevant RPSB forms in the following discussion.

High-level ab initio calculations. To interpret the experimental results, we have performed multi-state multi-reference perturbation theory excited-state calculations for the all-trans and single cis isomers of RPSB, using the XMCQDPT2 $2^{42} / \mathrm{SA}(2)-\mathrm{CASSCF}$ (12/12)/cc-pVDZ method. In Fig. 4a-d, $S_{1}$ relaxed geometry scans for rotation about three central double bonds in the all-trans and single cis chromophores are shown. We found that all-trans RPSB has small barriers that hinder rotation about the $\mathrm{C}_{9}=\mathrm{C}_{10}, \mathrm{C}_{11}=$ $\mathrm{C}_{12}$, and $\mathrm{C}_{13}=\mathrm{C}_{14}$ bonds and specifically that the barrier is the smallest (by a factor of 2.5) for rotation about the $\mathrm{C}_{11}=\mathrm{C}_{12}$ bond. For all-trans $\rightarrow 11$-cis isomerization, the barrier is $0.04 \mathrm{eV}$ and the corresponding minimum-energy conical intersection (MECI) lies $0.3 \mathrm{eV}$ below the minimum in $\mathrm{S}_{1}$, thus providing a direct route to the ground state after crossing the barrier in $S_{1}$. The associated excited-state lifetime of all-trans RPSB is calculated to be 4 ps at $300 \mathrm{~K}$ and as long as $92 \mathrm{ps}$ at $100 \mathrm{~K}$. These values are in agreement with the long time component observed in the present measurements, $3 \mathrm{ps}$ at $300 \mathrm{~K}$ and $77 \mathrm{ps}$ at $100 \mathrm{~K}$, which is thus assigned to the decay of $S_{1}$ in all-trans RPSB.

According to our calculations, the 9-cis, 11-cis, and 13-cis isomers exhibit essentially flat potential-energy surfaces around the corresponding minima in $S_{1}$ and hence undergo ultrafast, almost barrierless internal conversion from $S_{1}$ to $S_{0}$. The steepest pathway is found for the 11-cis isomer (Fig. 4b). This is consistent with our measured fast sub-ps component. However, this component also shows a temperature dependence and increases 


\section{a}

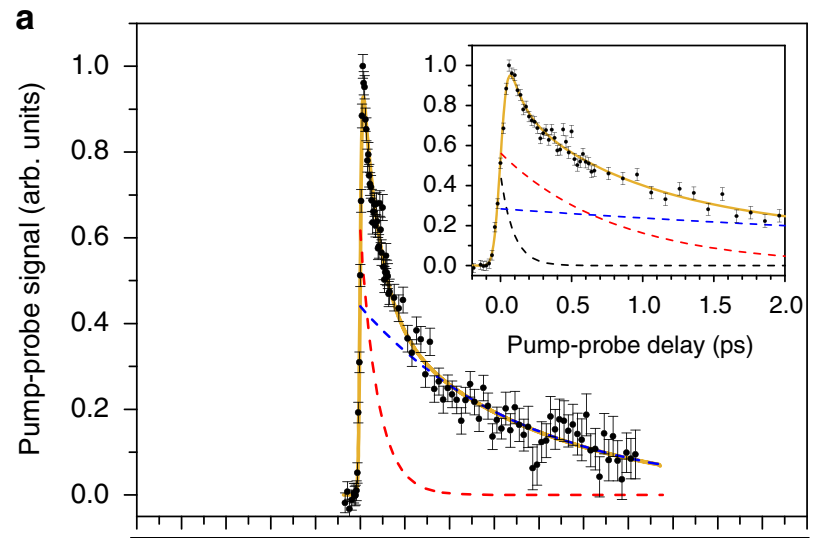

c

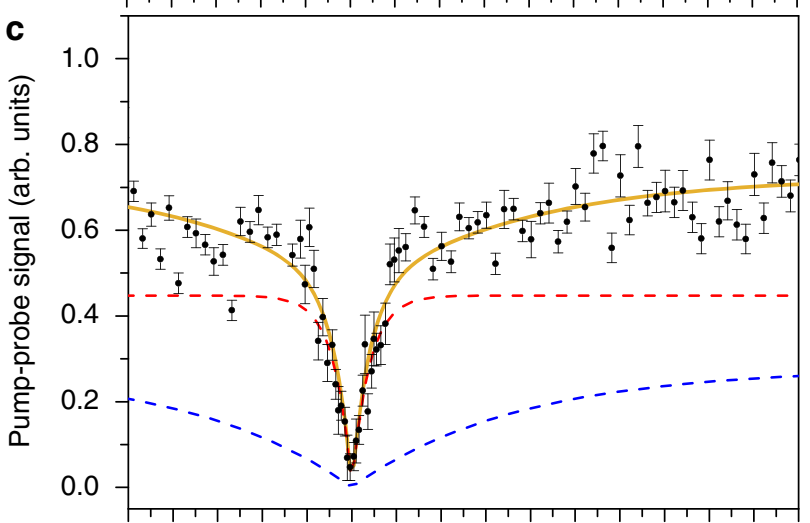

e

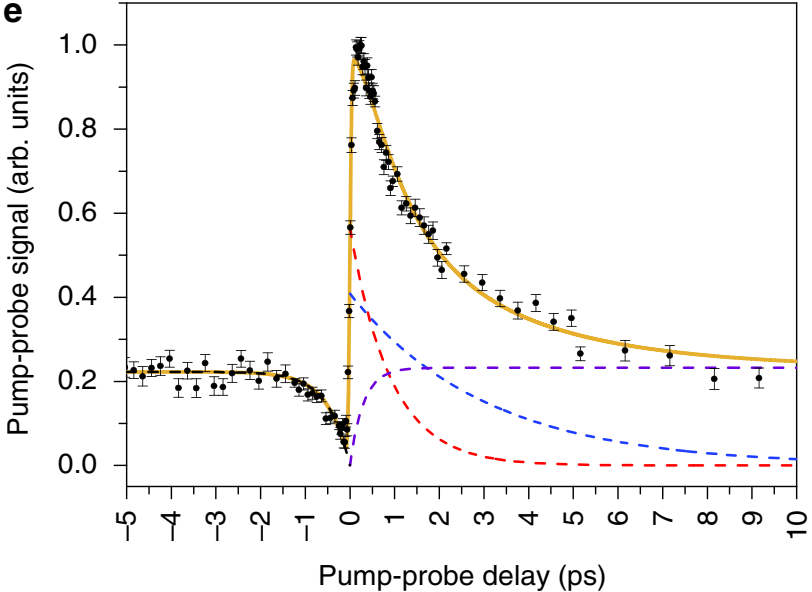

b

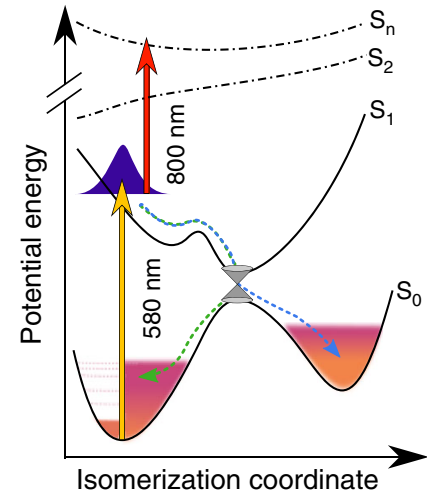

d $\hat{f} \ldots \ldots \ldots$

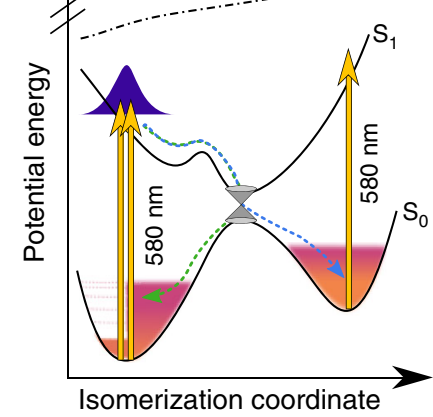

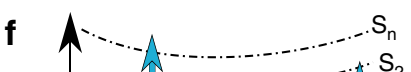

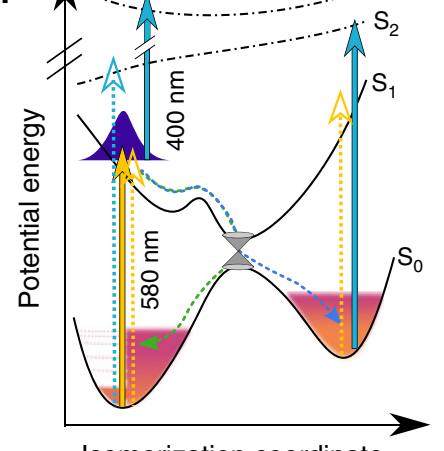

Isomerization coordinate

Fig. 2 Pump-probe measurements of retinal protonated Schiff-base (RPSB) in vacuo. a, b Excited-state decay seen as the two-photon, pump-probe signal as a function of delay between pump $(580 \mathrm{~nm})$ and probe $(800 \mathrm{~nm})$ pulses. The full curve is from a fit with two exponential functions folded with the $80 \mathrm{fs}$ cross-correlation in the experiment. The dashed lines represent the two decay components in the fit ( $395 \pm 42 \mathrm{fs}$ and $3.7 \pm 0.4 \mathrm{ps}$ ). The insert shows a fit with three lifetime components, which specifically takes into account the separate early sub $\sim 100 \mathrm{fs}$ dynamics (fitted lifetimes $70 \pm 20 \mathrm{fs}$, $785 \pm 185 \mathrm{fs}$, and $5.6 \pm 1.8$ ps). Data with $900 \mathrm{~nm}$ probe and other pump wavelengths within the $\mathrm{S}_{0} \rightarrow \mathrm{S}_{1}$ absorption band may be found in the Supplementary Table 1. c, d Ground-state recovery revealed by the two-photon, pump-probe signal as a function of delay between pump $(580 \mathrm{~nm})$ and probe ( $580 \mathrm{~nm})$ pulses. The full curve is a fit with the two lifetimes found from the decay of the excited state (shown in $\mathbf{a}$ ). e, $\mathbf{f}$ Dynamics revealed by the two-photon, pump-probe signal as a function of delay between pump $(580 \mathrm{~nm})$ and probe $(400 \mathrm{~nm})$ pulses. The chromophore may here absorb two photons (pump and probe) at all times, except right after excitation to $S_{2}$ by $400 \mathrm{~nm}$ at small negative pump-probe delays. The fast recovery on the negative time axis is associated with the return to $S_{0}$ after initial excitation $(400 \mathrm{~nm})$ to $S_{2}$. All data shown here are recorded at room temperature. All error bars presented are one standard deviation (SD)

from $400 \mathrm{fs}$ at $300 \mathrm{~K}$ to $1.4 \mathrm{ps}$ at $100 \mathrm{~K}$ (Fig. 3). Using the experimentally determined lifetime at the low temperature, we can estimate a barrier height for the cis isomers in $\mathrm{S}_{1}$. This gives a barrier which is approximately one order of magnitude lower than that found in all-trans RPSB. Such a small barrier height is consistent with the calculated flat potential-energy surface of the $\mathrm{S}_{1}$ state for the cis isomers, in particular for 9-cis and 13-cis RPSB. The almost barrierless photoisomerization in the gas phase documented here is indeed in agreement with CASSCF theory for the 11-cis isomer ${ }^{21}$.

The calculated $S_{1}-S_{n}$ vertical excitation energies along the $\mathrm{C}_{11}=\mathrm{C}_{12}$ isomerization coordinate of all-trans and 11-cis RPSB (Fig. 4e, f) show that the transient absorption (TA) corresponds to the $S_{1}-S_{3}$ excitation, which is the brightest transition along the entire reaction pathway. It shifts to shorter wavelengths as a vibrational wavepacket travels in the $S_{1}$ state towards the conical 


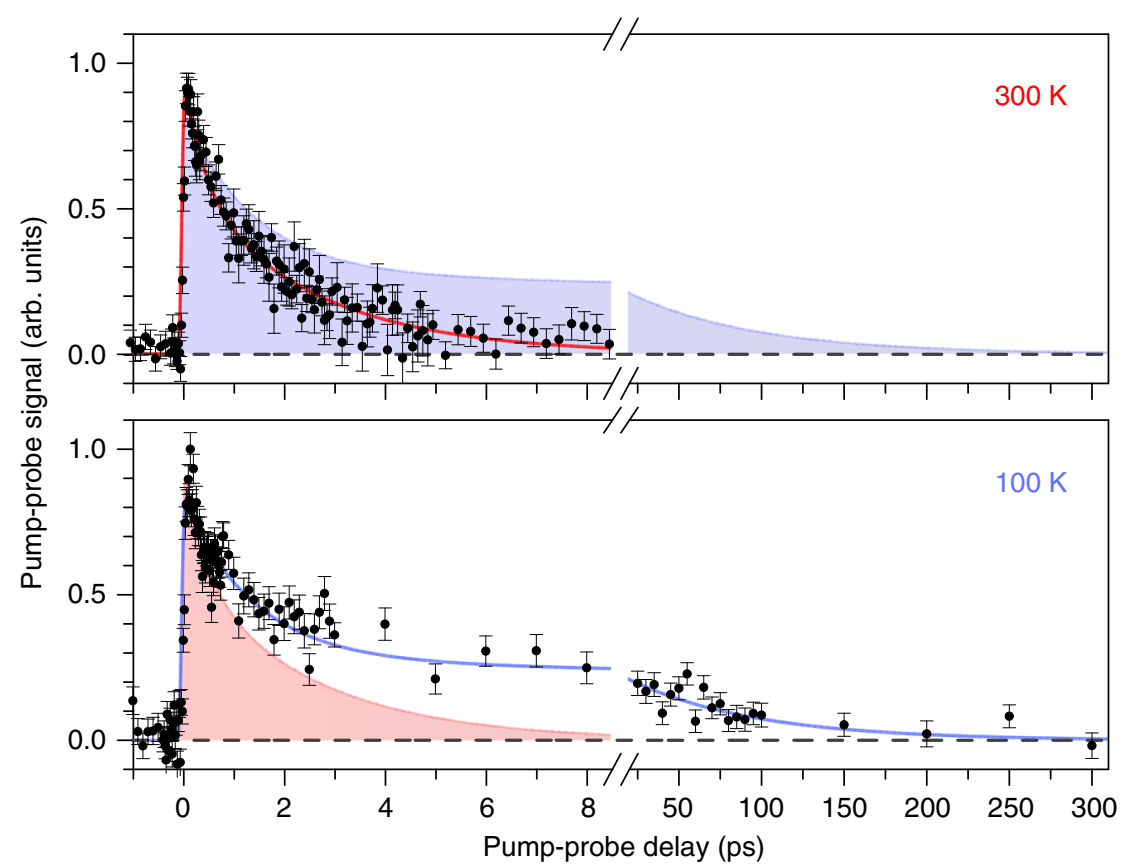

Fig. 3 Pump-probe signal as a function of the pump-probe delay at 300 and $100 \mathrm{~K}$. The excited-state decay of retinal protonated Schiff-base (RPSB) at two temperatures. Upper graph: Two-photon signal as a function of delay between pump ( $600 \mathrm{~nm})$ and probe $(800 \mathrm{~nm})$ pulses, recorded at $300 \mathrm{~K}$. Lifetimes of about $400 \mathrm{fs}$ and $3 \mathrm{ps}$ are here deduced. Lower graph: Same as upper graph, but recorded at $100 \mathrm{~K}$ where the lifetimes are 1.4 and $77 \mathrm{ps.} \mathrm{The} \mathrm{shaded} \mathrm{areas}$ are shown to provide a cross-comparison with the data obtained at the other temperature. All error bars presented are one standard deviation (SD)

intersection (Fig. 4g). Indeed, the enhanced experimental TA signals, seen at 720 and $660 \mathrm{~nm}$, match the bright $S_{1}-S_{3}$ vertical transitions at the optimized geometries of the $S_{1}$ minimum and transition state, respectively (see Fig. 5).

The $S_{2}$ state does not appreciably mix with $S_{1}$ along the reaction pathway as also shown by other methods like CASSCF ${ }^{21}$. The smallest energy gap is $0.9 \mathrm{eV}$ and steeply rising as the chromophore twists (see Fig. 4e, f). The mechanism of internal conversion is thus defined solely by the interplay between the ground and first excited states in RPSB, where the latter is dominated by a charge-transfer character, as also demonstrated recently for RPSB in the gas phase ${ }^{43}$.

The absence of a potential-energy barrier in the single cis isomers can be attributed to sterical hindrance imposed by the repulsive interaction of the methyl groups with the hydrogen atoms of the conjugated tail of the chromophore, resulting in destabilization of the planar equilibrium structures in $S_{1}$ and almost barrierless internal conversion in cis isomers. The equilibrium structures of 9-cis and 13-cis RPSB lie 0.09 and $0.12 \mathrm{eV}$ higher in energy compared to that of all-trans RPSB, respectively. In the 11-cis isomer, the $S_{1}$ planar structure lies $0.22 \mathrm{eV}$ higher in energy compared to that of all-trans RPSB and is not stable with respect to rotation about the $\mathrm{C}_{11}=\mathrm{C}_{12}$ bond at the XMCQDPT2/SA(2)-CASSCF $(12,12) / c c-p V D Z$ level of theory.

The fraction of ions in the short-lived component increases from 40 to $57 \%$ in going from 300 to $100 \mathrm{~K}$, thus indicating that the cis isomers are most likely formed through light exposure or collisions. The thermal backward cis $\rightarrow$ trans reaction in the ground state is strongly influenced by the temperature of the ions, hence resulting in a larger fraction of the cis isomers trapped in the ground state at low temperature. These fractions are also consistent with ion mobility spectrometry (IMS) measurements ${ }^{32,41}$. Furthermore, the all-trans $\rightarrow$ cis photoreaction shows specificity in the gas phase, as evidenced by our calculations, thus predominantly resulting in the 11-cis isomer.
Starting with an 11-cis sample, the decay is also described by two components, a short $650 \pm 160$ fs component and a long $4.7 \pm 3$ ps component at $300 \mathrm{~K}$ (see Fig. 6), similar to the dynamics of the alltrans sample. Here, the fraction of ions in the short-lived component is $78 \%(300 \mathrm{~K})$, supporting that the fast component is connected with the 11-cis isomer.

The excited-state relaxation dynamics of a recently discovered bacterial rhodopsin, KR2, which functions as a light-driven sodium-ion pump and contains all-trans RPSB shows several time constants ${ }^{44}$. A major $180 \mathrm{fs}$ component is attributed to the reactive decay of $S_{1}$, while two minor components of 3 and $30 \mathrm{ps}$ are assigned to nonreactive $S_{1}$ states. The decay by isomerization of $S_{1}$ occurs three times faster in KR2 than in bR, and hence the protein environment can indeed change the timescale of photoisomerization.

The mechanism that produces nonreactive $S_{1}$ states, also reported for other retinal-containing proteins ${ }^{44}$, is not clear. However, in light of our findings, it is tempting to assign the slow picosecond components observed in KR2 to the dynamics of all-trans RPSB in its native, planar conformation inside the protein, assuming that active-site structural heterogeneity involves different conformations of all-trans RPSB inside the chromophore-binding pocket. The protein may facilitate photoisomerization by changing the native conformation of all-trans RPSB through pre-twisting it in $S_{0}$, thus reducing the barrier height for double-bond rotation in $S_{1}$ and decreasing the timescale considerably. The slow 3 ps component found in KR2 is remarkably similar to that observed in the present study for the bare all-trans RPSB. Moreover, our calculated lifetimes for isomerization about $\mathrm{C}_{11}=\mathrm{C}_{12}$ and $\mathrm{C}_{13}=$ $\mathrm{C}_{14}$ (or $\mathrm{C}_{9}=\mathrm{C}_{10}$ ) bonds are $\sim 5$ and $\sim 40 \mathrm{ps}$, respectively. These values are to be compared with the slow 3 and 30 ps time constants of KR2. In both cases, the planar all-trans PSBR chromophore is slow and most likely not operational in the photocycle of the protein. Hence, the native planar conformation of the chromophore might be responsible for slow nonreactive dynamics in retinal-containing proteins. 
a<smiles>CCCC[NH+]=CC=C(C)C=CC=C(C)C=CC1=C(C)CCCC1(C)C</smiles>

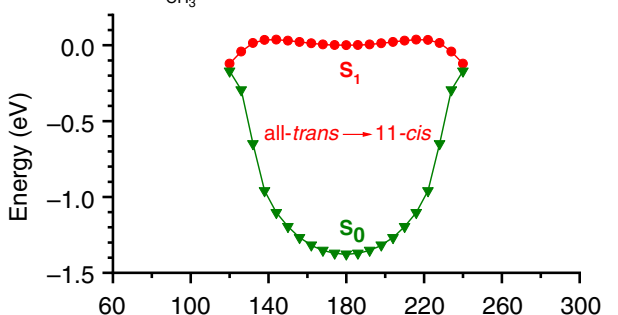

C
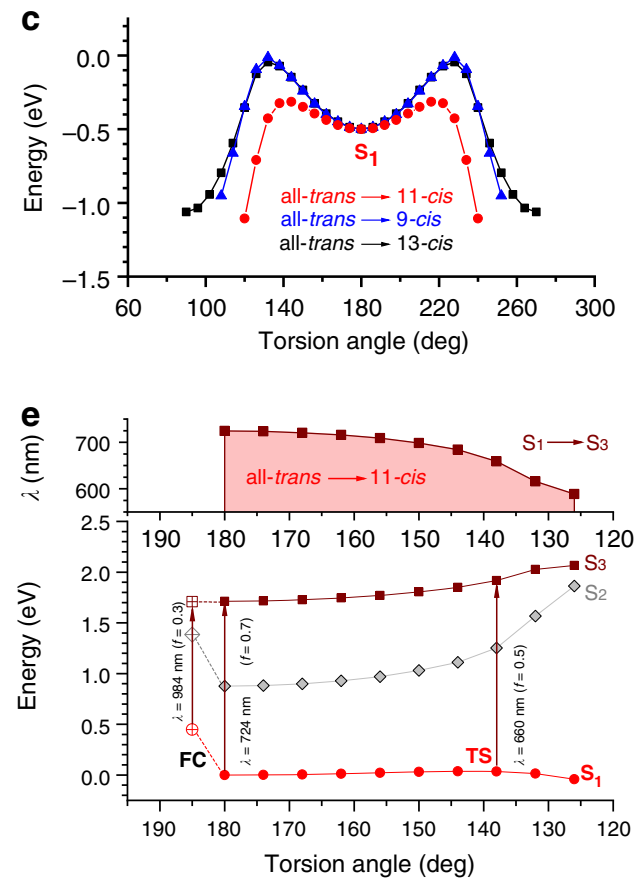

g

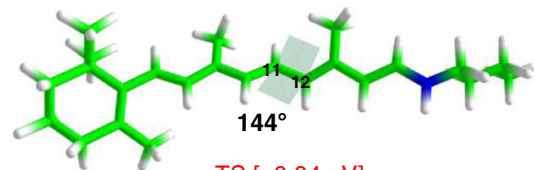

TS $[+0.04 \mathrm{eV}]$

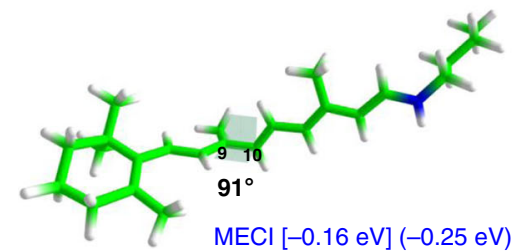

b

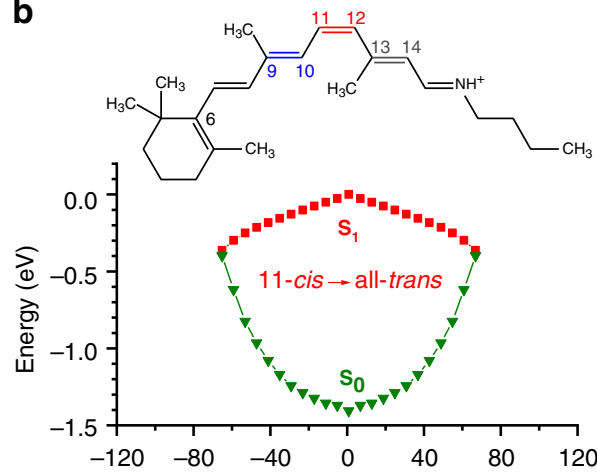

d
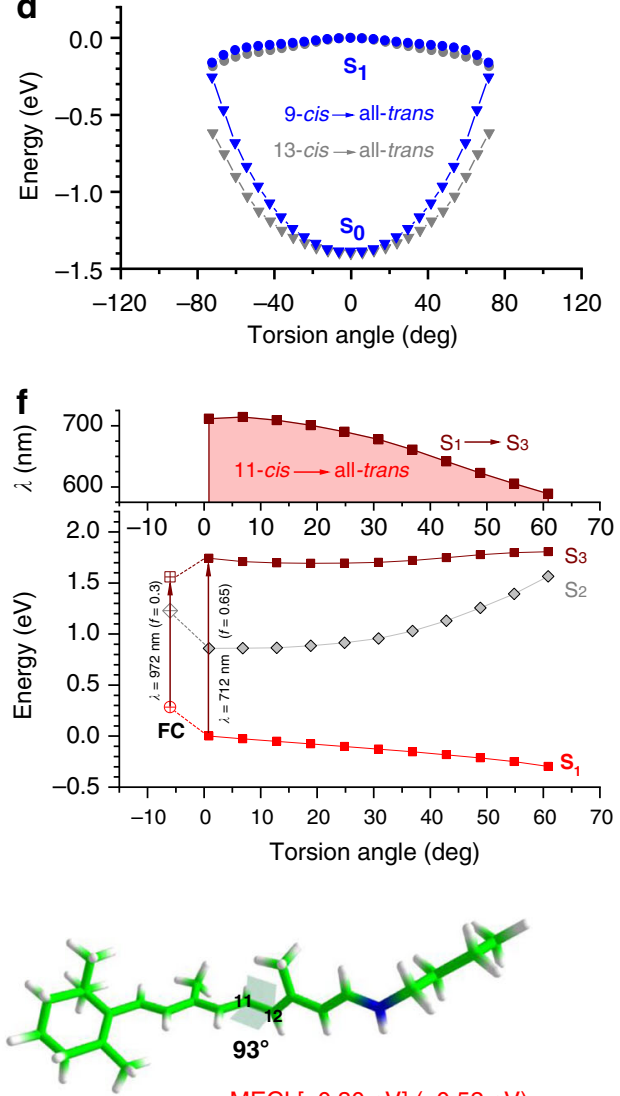

$\operatorname{MECl}[-0.30 \mathrm{eV}](-0.52 \mathrm{eV})$

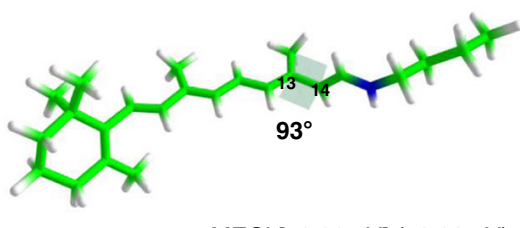

$\operatorname{MECl}[-0.08 \mathrm{eV}](-0.20 \mathrm{eV})$

Fig. 4 Calculated potential-energy curves along the isomerization coordinates in all-trans, 11-cis, 9-cis, and 13-cis retinal protonated Schiff-base (RPSB). a-d Excited-state relaxed geometry scans for rotation about double bonds are shown in red $\left(\angle \mathrm{C}_{10} \mathrm{C}_{11}=\mathrm{C}_{12} \mathrm{C}_{13}\right)$, blue $\left(\angle \mathrm{C}_{8} \mathrm{C}_{9}=\mathrm{C}_{10} \mathrm{C}_{11}\right)$, and black $\left(\angle C_{12} C_{13}=C_{14} C_{15}\right)$. Ground-state energies calculated at the $\mathrm{S}_{1}$ optimized geometries along the lowest-energy pathway are shown in green. The planar structures in $\mathrm{S}_{1}$ are obtained through the unconstrained optimization for all isomers, except for the 11-cis isomer, where the torsion angle is kept fixed. Note that the $6 \mathrm{~s}$-cis and $6 \mathrm{~s}$-trans rotomers are found to have the same barrier heights that hinder isomerization in $\mathrm{S}_{1}$ in all-trans RPSB (see Supplementary Figure 4). e, f Transient absorption calculated as vertical excitation energies $S_{1}-S_{3}$ in all-trans and 11-cis RPSB. Oscillator strengths $f$ along the reaction pathways are shown in brackets. Note that the $S_{1}-S_{2}$ transition has a lower oscillator strength of $<0.1$ and gains some intensity $(f=0.2)$ through mixing with the $S_{3}$ state near the Franck-Condon ( $F C$ ) point and closer to the $S_{1} / S_{0}$ conical intersections. $\mathbf{g}$ Optimized structures of the lowest-energy transition state in $\mathrm{S}_{1}$ in all-trans RPSB (TS) and the minimum-energy conical intersections $\mathrm{S}_{1} / \mathrm{S}_{0}(\mathrm{MECI})$. Carbon atoms are depicted in green, hydrogen atoms in white, and the nitrogen atom in blue. Energies in square and round brackets are shown with respect to the all-trans and the corresponding single cis RPSB planar structures in $\mathrm{S}_{1}$, respectively 

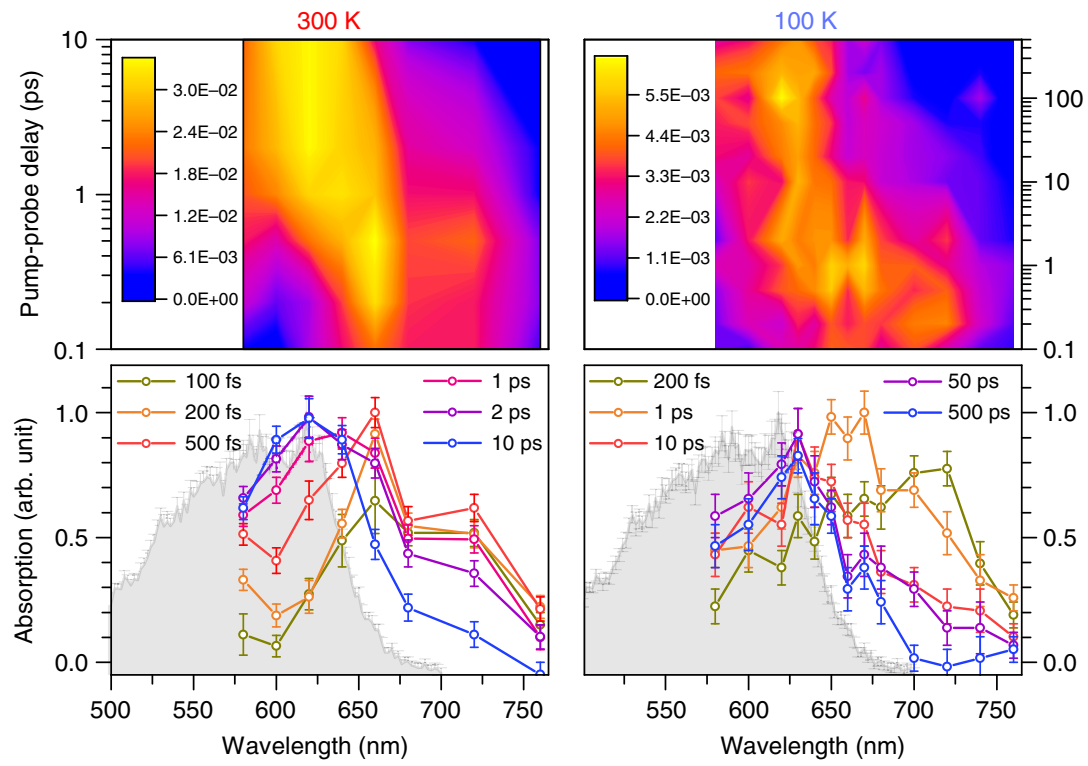

Fig. 5 Transient absorption (TA) measurements of retinal protonated Schiff-base (RPSB) in the gas phase at 300 and $100 \mathrm{~K}$. The TA data are shown as a function of variable probe wavelength (fixed $600 \mathrm{~nm}$ pump). The grey area shows the ground-state absorption profile at $300 \mathrm{~K}$ (one-photon spectrum). The transient absorption spectra at asymptotic long times (10-500 ps) correspond to absorption from a hot ground state, peaking at $620 \mathrm{~nm}$. The excitedstate absorption in the red part disappears with time concomitantly with a rise of the ground-state absorption with a lifetime, which strongly depends on temperature. Three major peaks at 720,660 , and $620 \mathrm{~nm}$ are seen at both temperatures. See discussion in the text

\section{Discussion}

The excited-state dynamics of RPSB-containing proteins as well as of RPSB in solutions shows a variety of time constants, typically in the sub-ps and few-ps regime. We have studied, for the first time, the dynamics of the bare RPSB chromophore in vacuo and found timescales of the very same order. When excited to the first electronically excited state, the decay is found to be triexponential, where the two short lifetimes together are represented by a $\sim 400 \mathrm{fs}$ decay. This decay time is independent of the excitation wavelength, but temperature dependent, raising by a factor of about three when the RPSB chromophore is cooled to $100 \mathrm{~K}$. It gives evidence that a barrier as small as $30 \mathrm{~cm}^{-1}$ is operative in internal conversion for the bare chromophore. A longer, several picosecond component is also involved. This component reaches a decay time of almost 100 ps when cooled to $100 \mathrm{~K}$. According to our calculations this is consistent with a $0.04 \mathrm{eV}\left(\sim 300 \mathrm{~cm}^{-1}\right)$ energy barrier in $\mathrm{S}_{1}$ for all-trans RPSB. Experimentally and theoretically the long picosecond decay is thus assigned to RPSB in the all-trans form. Theoretically, we find the isomerization of RPSB in the 11-cis form to be essentially barrierless in $S_{1}$ and the short sub-picosecond component may therefore be ascribed to 11-cis RPSB. Based on our findings, we conclude that the visual photoreceptors rely on the ultrafast intrinsic photoresponse of their 11-cis chromophore, whereas bacterial rhodopsins tune the photoresponse of the all-trans RPSB chromophore by particularly reducing the barrier height about the $\mathrm{C}_{13}=\mathrm{C}_{14}$ double bond, thus changing both the timescale and specificity of photoisomerization of RPSB compared to those found in the intrinsic photoresponse, where the lowest barrier is for rotation about the $\mathrm{C}_{11}=\mathrm{C}_{12}$ double bond.

\section{Methods}

Experimental setup. The experiments were performed at the SAPHIRA electrostatic ion-storage ring ${ }^{38,45}$, where an electrospray ion source with a multipole ion trap was used to produce bunches of bio-chromophores in vacuo.

Ion source and sample preparation. All-trans as well as 11-cis Schiff-base retinal chromophores were dissolved in methanol and electrosprayed as protonated ions from a steel needle. Solvent molecules were evaporated off in the capillary, which was heated to about $100^{\circ} \mathrm{C}$, resulting in bare, singly charged molecules, which were transported through an octopole ion guide to a 16-pole radio frequency ion trap. Helium buffer-gas collisions reduced the translational kinetic energy causing the ions to be trapped. The ions were thermalized to the buffer-gas temperature (100 or $300 \mathrm{~K})$ while trapped. At $100 \mathrm{~Hz}$ repetition rate, ions were extracted as $20 \mu$ s bunches. The ion source was located on a $4 \mathrm{kV}$ high-voltage platform, which provided extraction from the source and subsequent acceleration to $4 \mathrm{keV}$. The retinal ions were mass-tocharge selected through a dipole magnet prior to injection into SAPHIRA.

Ion storage and detection. SAPHIRA has four quadrupole $90^{\circ}$ bending electrodes mounted with quadrupole triplet lenses at the entrance and exit of each corner to keep the ion beam collimated in the ring. Two particle detectors mounted at the end of corner 3 and 4 detected neutrals generated by residual gas collisions (background pressure $2.0 \mathrm{E}-8 \mathrm{mbar}$ and from photo-induced dissociation, see Supplementary Figure 1).

Laser system and optical setup. Laser pulses were generated by a Ti:Sapphirebased Libra HE-UFS oscillator and regenerative amplifier system, delivering $4 \mathrm{~mJ}$, $50 \mathrm{fs}$ pulses with $1 \mathrm{kHz}$ repetition rate. The pulses were split 3:1 to separately pump two TOPAS-C optical parametric amplifiers, which generated the pump and probe pulses. One of the beams was delayed by a motorized delay stage. The two beams were collinearly overlapped using a beam splitter or dichroic mirror, depending on the pump and probe wavelength. The cross-correlation of two $580 \mathrm{~nm}$ pulses was measured to be $80 \mathrm{fs}$ in a $\beta$-barium borate crystal by sum-frequency generation. The pulse energies were $100 \mu \mathrm{J}$ at $580 \mathrm{~nm}, 60 \mu \mathrm{J}$ at $610 \mathrm{~nm}$, and $200 \mu \mathrm{J}$ at $400 \mathrm{~nm}$.

Typical experimental cycle. The ion bunches circulated for about $2 \mathrm{~ms}$ in SAPHIRA prior to laser excitation. The first laser pulse excited the retinal chromophores from the $S_{0}$ ground state to the $S_{1}$ excited state. Internal conversion brought the ions back into the ground state, now with significantly increased internal energy, leading to fragmentation on the millisecond timescale. The total pump-probe signal appears from the increased fragmentation of both pump and probe photon absorption. For the final data analysis, we consider only the counts of the first pump-probe signal peak (as a function of the pump-probe delay), to limit statistical fluctuations from background subtraction. Error bars are estimated considering only the statistics of the neutral counts.

Computational methods. All energy and gradient calculations in both excited and ground states are performed using the extended multiconfiguration quasidegenerate perturbation theory, XMCQDPT $2^{42}$. Structures of the stationary points in $S_{1}$ and the $S_{1} / S_{0}$ MECIs, as well as relaxed geometry potential-energy scans along isomerization coordinates in $S_{1}$ are obtained at the XMCQDPT2 $2 / S A(2)$-CASSCF $(12,12) / c c-p V D Z$ level of theory. Vertical excitation energies $S_{1}-S_{n}$ along reaction pathways are calculated at the XMCQDPT2[7]/SA(7)-CASSCF(12,12)/cc-pVDZ level of theory. The Firefly package ${ }^{46}$, version 8.2 .0 , is used for all electronic 

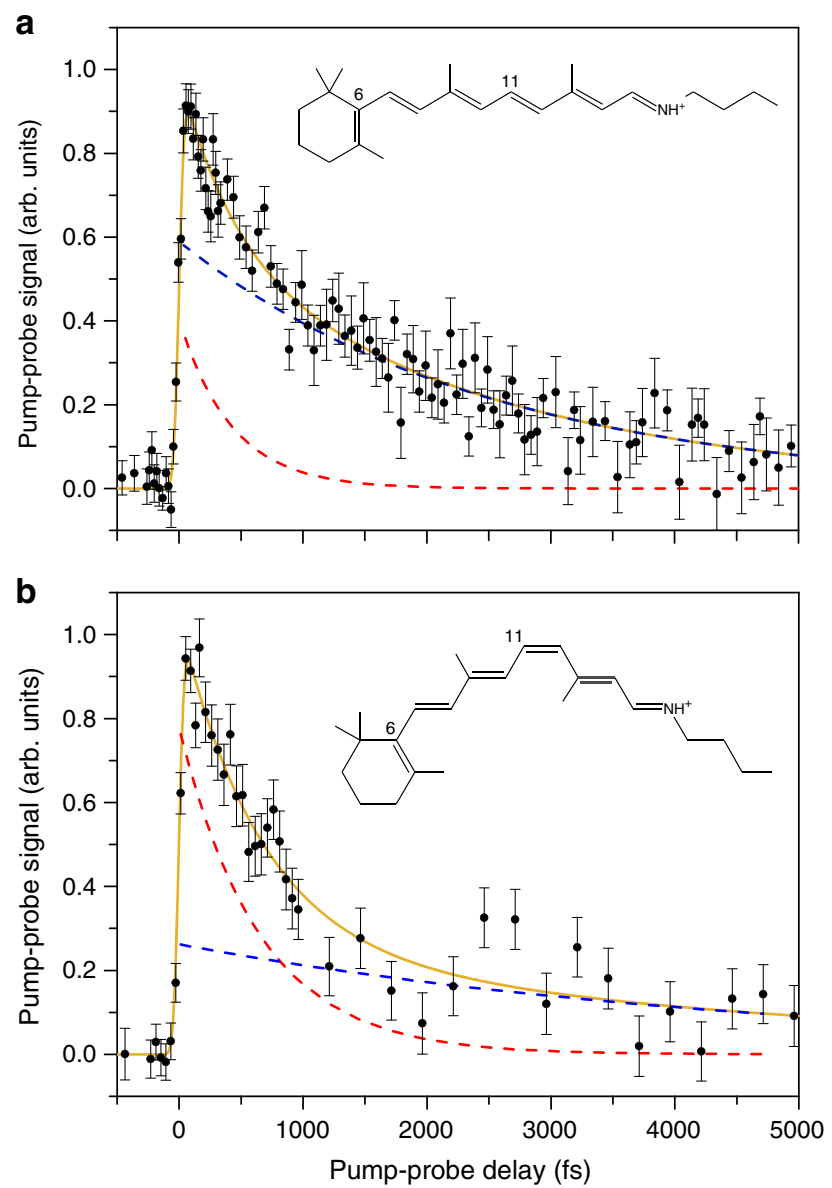

Fig. 6 Pump-probe signal for all-trans and 11-cis retinal protonated Schiffbase (RPSB) samples in the gas phase. Pump-probe signal for all-trans (top) and 11-cis (bottom) samples obtained with $600 \mathrm{~nm}$ pump and $800 \mathrm{~nm}$ probe at room temperature. The full curve is from a fit with two exponential functions folded with the $80 \mathrm{fs}$ cross-correlation in the experiment. The dashed lines represent the two decay components in the fit $424 \pm 121 \mathrm{fs}$ and $2.5 \pm 0.3 \mathrm{ps}$ for all-trans and $650 \pm 160 \mathrm{fs}$ and $4.7 \pm 3$ ps for 11 -cis RPSB. All error bars presented are one standard deviation (SD)

structure calculations. Excited-state lifetimes of all-trans RPSB are calculated using transition state theory as an inverse of a sum of rate constants that refer to rotation about three double bonds. We assume the same thermal population of vibrational levels in $S_{1}$ as in $S_{0}$, following excitation that is close to the adiabatic transition in RPSB. An excess of vibrational energy gained upon excitation is therefore considered to be small compared to an average vibrational energy of the chromophore ions equilibrated in the ground state prior to excitation. Partition functions for the reaction modes are calculated based on the exact eigenvalues found as numerical solutions to the one-dimensional Schrödinger equation using the calculated XMCQDPT2 potentials that hinder rotation about double bonds in $S_{1}$.

\section{Data availability}

The authors declare that the data supporting the findings of this study are available from the corresponding author upon reasonable request.

Received: 31 August 2018 Accepted: 27 February 2019

Published online: 14 March 2019

\section{References}

1. Stoeckenius, W. \& Bogomolni, R. A. Bacteriorhodopsin and related pigments of halobacteria. Annu. Rev. Biochem. 51, 587-616 (1982).

2. Venter, J. C. et al. Environmental genome shotgun sequencing of the sargasso sea. Science 304, 66-74 (2004).
3. Mathies, R., Brito Cruz, C., Pollard, W. \& Shank, C. Direct observation of the femtosecond excited-state cis-trans isomerization in bacteriorhodopsin. Science 240, 777-779 (1988).

4. Hamm, P. et al. Femtosecond spectroscopy of the photoisomerisation of the protonated Schiff base of all-trans retinal. Chem. Phys. Lett. 263, 613-621 (1996).

5. Logunov, S. L. \& El-Sayed, M. A. Redetermination of the quantum yield of photoisomerization and energy content in the k-intermediate of bacteriorhodopsin photocycle and its mutants by the photoacoustic technique. J. Phys. Chem. B 101, 6629-6633 (1997).

6. Logunov, S. L., Song, L. \& El-Sayed, M. A. Excited-state dynamics of a protonated retinal Schiff base in solution. J. Phys. Chem. 100, 18586-18591 (1996).

7. Kandori, H. \& Sasabe, H. Excited-state dynamics of a protonated Schiff base of all-trans retinal in methanol probed by femtosecond fluorescence measurement. Chem. Phys. Lett. 216, 126-172 (1993).

8. Freedman, K. A. \& Becker, R. S. Comparative investigation of the photoisomerization of the protonated and unprotonated $n$-butylamine Schiff bases of 9-cis-, 11-cis-, 13-cis-, and all-trans-retinals. J. Am. Chem. Soc. 108, 1245-1251 (1986).

9. Koyama, Y., Kubo, K., Komori, M., Yasuda, H. \& Mukai, Y. Effect of protonation on the isomerization properties of n-butylamine Schiff base of isomeric retinal as revealed by direct hplc analyses: selection of isomerization pathways by retinal proteins. Photochem. Photobiol. 54, 433-443 (1991).

10. Toker, Y. et al. Photoresponse of the protonated Schiff-base retinal chromophore in the gas phase. Phys. Chem. Chem. Phys. 15, 19566-19569 (2013).

11. Coughlan, N. J. A., Catani, K. J., Adamson, B. D., Wille, U. \& Bieske, E. J. Photoisomerization action spectrum of retinal protonated Schiff base in the gas phase. J. Chem. Phys. 140, 164307 (2014).

12. Fernald, R. D. Casting a genetic light on the evolution of eyes. Science 313, 1914-1918 (2006).

13. Schoenlein, R., Peteanu, L., Mathies, R. \& Shank, C. The first step in vision: femtosecond isomerization of rhodopsin. Science 254, 412-415 (1991).

14. Peteanu, L. A., Schoenlein, R. W., Wang, Q., Mathies, R. A. \& Shank, C. V. The first step in vision occurs in femtoseconds: complete blue and red spectral studies. Proc. Natl Acad. Sci. USA 90, 11762-11766 (1993).

15. Haran, G., Morlino, E. A., Matthes, J., Callender, R. H. \& Hochstrasser, R. M. Femtosecond polarized pump-probe and stimulated emission spectroscopy of the isomerization reaction of rhodopsin. J. Phys. Chem. A 103, 2202-2207 (1999).

16. Kandori, H. et al. Excited-state dynamics of rhodopsin probed by femtosecond fluorescence spectroscopy. Chem. Phys. Lett. 334, 271-276 (2001).

17. Wand, A., Gdor, I., Zhu, J., Sheves, M. \& Ruhman, S. Shedding new light on retinal protein photochemistry. Annu. Rev. Phys. Chem. 64, 437-458 (2013).

18. Johnson, P. J. M. et al. Local vibrational coherences drive the primary photochemistry of vision. Nat. Chem. 7, 980 (2015)

19. Polli, D. et al. Conical intersection dynamics of the primary photoisomerization event in vision. Nature 467, 440-443 (2010).

20. Schapiro, I. et al. The ultrafast photoisomerizations of rhodopsin and bathorhodopsin are modulated by bond length alternation and HOOP driven electronic effects. J. Am. Chem. Soc. 133, 3354-3364 (2011).

21. Rivalta, I., Nenov, A. \& Garavelli, M. Modelling retinal chromophores photoisomerization: from minimal models in vacuo to ultimate bidimensional spectroscopy in rhodopsins. Phys. Chem. Chem. Phys. 16, 16865-16879 (2014).

22. Garavelli, M., Negri, F. \& Olivucci, M. Initial excited-state relaxation of the isolated 11-cis protonated Schiff base of retinal: evidence for in-plane motion from $\mathrm{ab}$ initio quantum chemical simulation of the resonance raman spectrum. J. Am. Chem. Soc. 121, 1023-1029 (1999).

23. Punwong, C., Owens, J. \& Martínez, T. J. Direct QM/MM excited-state dynamics of retinal protonated Schiff base in isolation and methanol solution. J. Phys. Chem. B 119, 704-714 (2014).

24. Becker, R. S. \& Freedman, K. A comprehensive investigation of the mechanism and photophysics of isomerization of a protonated and unprotonated Schiff base of 11-cis-retinal. J. Am. Chem. Soc. 107, 1477-1485 (1985).

25. Kandori, H., Katsuta, Y., Ito, M. \& Sasabe, H. Femtosecond fluorescence study of the rhodopsin chromophore in solution. J. Am. Chem. Soc. 117, 2669-2670 (1995).

26. Bassolino, G. et al. Barrierless photoisomerization of 11-cis retinal protonated Schiff base in solution. J. Am. Chem. Soc. 137, 12434-12437 (2015).

27. Zgrablić, G., Novello, A. M. \& Parmigiani, F. Population branching in the conical intersection of the retinal chromophore revealed by multipulse ultrafast optical spectroscopy. J. Am. Chem. Soc. 134, 955-961 (2011).

28. Sovdat, T. et al. Backbone modification of retinal induces protein-like excited state dynamics in solution. J. Am. Chem. Soc. 134, 8318-8320 (2012).

29. Bassolino, G. et al. Synthetic control of retinal photochemistry and photophysics in solution. J. Am. Chem. Soc. 136, 2650-2658 (2014). 
30. Liu, L., Liu, J. \& Martinez, T. J. Dynamical correlation effects on photoisomerization: Ab initio multiple spawning dynamics with MS-CASPT2 for a model trans-protonated Schiff base. J. Phys. Chem. B 120, 1940-1949 (2016).

31. Andersen, L. H. et al. Absorption of Schiff-base retinal chromophores in vacuo. J. Am. Chem. Soc. 127, 12347-12350 (2005).

32. Coughlan, N. J. A., Adamson, B. D., Gamon, L., Catani, K. \& Bieske, E. J. Retinal shows its true colours: photoisomerization action spectra of mobilityselected isomers of the retinal protonated schiff base. Phys. Chem. Chem. Phys. 17, 22623-22631 (2015).

33. Knudsen, J. L., Kluge, A., Bochenkova, A. V., Kiefer, H. V. \& Andersen, L. H. The UV-visible action-absorption spectrum of all-trans and 11-cis protonated Schiff base retinal in the gas phase. Phys. Chem. Chem. Phys. 20, 7190-7194 (2018).

34. Delori, F. C. et al. In vivo fluorescence of the ocular fundus exhibits retinal pigment epithelium lipofuscin characteristics. Investig. Ophthalmol. Vis. Sci. 36, 718 (1995).

35. Zgrablić, G., Voïtchovsky, K., Kindermann, M., Haacke, S. \& Chergui, M. Ultrafast excited state dynamics of the protonated Schiff base of all-trans retinal in solvents. Biophys. J. 88, 2779-2788 (2005).

36. Svendsen, A., Kiefer, H. V., Pedersen, H. B., Bochenkova, A. V. \& Andersen, L. $\mathrm{H}$. Origin of the intrinsic fluorescence of the green fluorescent protein. J. Am. Chem. Soc. 139, 8766-8771 (2017).

37. Kiefer, H. V., Pedersen, H. B., Bochenkova, A. V. \& Andersen, L. H. Decoupling electronic versus nuclear photoresponse of isolated green fluorescent protein chromophores using short laser pulses. Phys. Rev. Lett. 117, 243004 (2016).

38. Pedersen, H. B. et al. Characterization of a new electrostatic storage ring for photofragmentation experiments. Rev. Sci. Instrum. 86, 063107 (2015).

39. Andersen, L. H. et al. Experimental studies of the photophysics of gas-phase fluorescent protein chromophores. Phys. Chem. Chem. Phys. 6, 2617-2627 (2004).

40. Goran, Z., Haacke, S. \& Chergui, M. Heterogeneity and relaxation dynamics of the photoexcited retinal Schiff base cation in solution. J. Phys. Chem. B 113, 4384-4393 (2009).

41. Dilger, J. et al. Direct measurement of the isomerization barrier of the isolated retinal chromophore. Angew. Chem. Int. Ed. 54, 4748-4752 (2015).

42. Granovsky, A. A. Extended multi-configuration quasi-degenerate perturbation theory: the new approach to multi-state multi-reference perturbation theory. $J$. Chem. Phys. 134, 214113 (2011).

43. Toker, Y. et al. Counterion-controlled spectral tuning of the protonated Schiffbase retinal. Phys. Rev. A 98, 043428 (2018).

44. Tahara, S. et al. Ultrafast photoreaction dynamics of a light-driven sodiumion-pumping retinal protein from krokinobacter eikastus revealed by femtosecond time-resolved absorption spectroscopy. J. Phys. Chem. Lett. 6, 4481-4486 (2015).

45. Svendsen, A., Teiwes, R., Kiefer, H. V., Andersen, L. H. \& Pedersen, H. B. Analysis of ionic photofragments stored in an electrostatic storage ring. Rev. Sci. Instrum. 87, 013111 (2016).

46. Granovsky, A. A. Firefly. http://classic.chem.msu.su/gran/firefly/index.html.

\section{Acknowledgements}

This work was supported by the Villum foundation as a Villum Experiment, project no. 17512 and the Carlsberg Foundation. A.V.B. and P.A.K. acknowledge support from the Russian Science Foundation (17-13-01276). The research is carried out using the equipment of the shared research facilities of HPC computing resources at Lomonosov Moscow State University. We thank Oriol Vendrell, Stefan Haacke, and Mudi Sheves for discussions and Henrik B. Pedersen for support with the SAPHIRA ion-storage ring.

\section{Author contributions}

L.H.A. conceived the 2D action-spectroscopy technique, and L.H.A. and H.V.K. designed the experiment together. E.G., H.V.K., J.L. and L.H.A. performed the measurements. E.G. and H.V.K. analyzed the experimental data. A.V.B. and P.A.K. performed ab initio calculations. L.H.A. and A.V.B. wrote the paper after discussions with all authors.

\section{Additional information}

Supplementary Information accompanies this paper at https://doi.org/10.1038/s41467 019-09225-7.

Competing interests: The authors declare no competing interests.

Reprints and permission information is available online at http://npg.nature.com/ reprintsandpermissions/

Journal peer review information: Nature Communications thanks the anonymous reviewers for their contribution to the peer review of this work.

Publisher's note: Springer Nature remains neutral with regard to jurisdictional claims in published maps and institutional affiliations.

cc (i) Open Access This article is licensed under a Creative Common Attribution 4.0 International License, which permits use, sharing, adaptation, distribution and reproduction in any medium or format, as long as you give appropriate credit to the original author(s) and the source, provide a link to the Creative Commons license, and indicate if changes were made. The images or other third party material in this article are included in the article's Creative Commons license, unless indicated otherwise in a credit line to the material. If material is not included in the article's Creative Commons license and your intended use is not permitted by statutory regulation or exceeds the permitted use, you will need to obtain permission directly from the copyright holder. To view a copy of this license, visit http://creativecommons.org/ licenses/by/4.0/.

(c) The Author(s) 2019 